\title{
Active Singularities for Multivehicle Motion Planning in an N-Vortex System
}

\author{
Francis D. Lagor ${ }^{1}$ and Derek A. Paley ${ }^{1,2}$ \\ 1 Department of Aerospace Engineering and Institute for Systems Research, \\ University of Maryland, College Park, MD, USA \\ 2 dpaley@umd.edu
}

\begin{abstract}
This paper presents a path-planning paradigm for distributed control of multiple sensor platforms in a geophysical flow well-approximated by a point-vortex model. We utilize Hamiltonian dynamics to generate control vector fields for vehicle motion in $N$-vortex flows using the concept of an active singularity whose strength is a tunable control input. We introduce active singularities that are virtual point vortices possibly collocated with virtual point sources or sinks. We provide a principled method to stabilize relative equilibria of these virtual vortices in the presence of the actual point vortices, which represent the underlying geophysical flow. We illustrate how these relative equilibria may be useful for vehicle path planning and sampling in a geophysical flow. Preliminary results presented here are based on an adaptive control design.
\end{abstract}

Keywords: path planning · vortex dynamics · environmental sampling - cooperative control

\section{Introduction}

Distributed environmental sampling is an active field of research [2],[14] due to its many applications, including contaminant plume localization [23], biological monitoring [18], and data assimilation in atmospheric and ocean sciences [5],[13]. Significant hardware and sensor improvements [19] as well as algorithmic performance guarantees [9],[10] have further encouraged interest. However, there are open challenges about how mobile sensor platforms can most effectively sample and interact with strong, circulating flows [3],[10],[11].

Coherent vortices (eddies) in the ocean persist on mesoscales (10 to $500 \mathrm{~km}$ ) and submesoscales (1 to $10 \mathrm{~km}$ ) for weeks or even months and play an important role in global transport and mixing processes [8]. For example, small-scale (15 $\mathrm{km}$ ) eddies appeared at the mouth of Monterey Bay during field experiments of the second Autonomous Ocean Sampling Network (AOSN-II) and contributed to the overall transport of cold water away from the southern part of the bay [18]. However, the movement of these eddies could not be correlated with local wind shear stress [18]. Understanding transport within these flow structures is possible but requires in situ observations over a large spatiotemporal volume collected by fleets of autonomous vehicles $[3],[6],[8],[17]$. Underwater gliders, which 
are steered, buoyancy-driven, long-endurance vehicles, and drifters, which are depth-controlled underwater platforms that passively drift, are deployed to reduce uncertainties in estimates of ocean processes [14],[16],[20] and to sample and track oceanographic features [2],[18]. A steered sampling platform such as a glider that travels within the flowfield of an eddy taking targeted observations may be even more beneficial than passive drifter, even if the drifter has longer endurance.

Difficulties in accessing platforms during relatively long ocean-sampling missions has encouraged research in distributed algorithms that can maximize vehicle endurance, enhance vehicle autonomy, and reduce process uncertainty during sampling. Hsieh et al. [6] show that steering vehicles to flow patterns such as (almost) invariant sets can help maximize vehicle endurance. Frew et al. [4] construct Lyapunov-based guidance vector fields for specifying sampling trajectories that can be tracked by the onboard controller of an autonomous (aerial) vehicle. Further, focusing measurements on targeted areas [6] and stabilizing multivehicle formations have been shown to help maximize information collection [3] when sampling geophysical flows that can be modeled using reduced-order methods such as a point-vortex model.

This paper provides a theoretically-justified motion-planning framework that accounts for environmental flow effects and vehicle-to-vehicle interactions through the use of flow singularities. Flow singularities (point vortices, sources, and sinks) are standard elements of a reduced-order potential flow model that induce nearby fluid flow but are undefined (singular) at their centers. Distributed algorithms within this framework are designed to enhance autonomy and to allow sampling platforms to exploit, whenever possible, the underlying motion of their environment to maximize endurance in geophysical flows.

The study of vortex dynamics in this paper is an extension of the fundamental point-vortex work of Chen et al. [1], who show that augmenting a point-vortex system with dissipation stabilizes relative equilibria (regular patterns that are fixed points in a reduced configuration space) of the unmodified point-vortex dynamics [1]. The modification of the point-vortex dynamics to include dissipation is suggestive of control action being applied to the point-vortex system. Further, we observe that the modified vortex dynamics in [1] represent spiral vortices (vortices collocated with a sources or sinks) in the presence of a sink at the origin. These observations lead us to consider spiral vortices as basic modeling elements of a virtual singularity system in which virtual vortices are used to guide vehicle sampling trajectories. By manipulating the virtual vortex strengths, we create active singularities to generate artificial vector fields for vehicle guidance. The actual vortex dynamics (representing the geophysical flow) and the virtual vortex dynamics are connected via a one-way influence of actual vortices on the virtual ones.

A point-vortex system possesses Hamiltonian dynamics and exhibits conservation of certain physical properties that we exploit in Lyapunov analysis to obtain analytical guarantees for this motion-planning paradigm. We provide Lyapunov stability analysis for the vortex dynamics of Chen et al. [1] based 
on conserved quantities of the Hamiltonian system. We extend the dynamics of Chen et al. [1] by showing how the location of the center of the relative equilibrium can be prescribed. We derive the total Hamiltonian, which is conserved by the virtual vortices in the actual-plus-virtual vortex dynamics, and use it to provide Lyapunov analysis demonstrating asymptotic convergence of virtual vortices to a relative equilibrium. The use of Lyapunov analysis to inform the selection of a parameter update law is a common theme in adaptive control [22]. As a preliminary example of the efficacy of this motion planning framework, we employ adaptive control to regulate the value of the total Hamiltonian.

The specific contributions of this paper are (1) Lyapunov analysis of the nonlinear stability of a relative equilibrium in the dissipative point-vortex system introduced by Chen et al. [1]; (2) a novel method for multivehicle motion planning in the presence of point vortices based on distributed control of active singularities; and (3) an adaptive control law for stabilizing lattice-shaped formations of sampling platforms around the actual center of vorticity in a point vortex flow. This work represents a framework in which multivehicle motion planning is achieved in the presence of idealized flow field dynamics.

The outline of the paper is as follows. Section II explains point vortex dynamics, summarizes the work of Chen et al. [1] on relative equilibrium configurations, and provides Lyapunov analysis of their model. Section III introduces active singularities for multivehicle motion planning and provides a Lyapunov analysis of relative-equilibrium stabilization for virtual vortices. Section IV presents a Lyapunov-based, distributed control strategy for formations of virtual vortices based on adaptive control of the singularity strength, including numerical simulation of motion planning in the presence of an actual vortex pair. Section V summarizes the paper and ongoing research.

\section{Point-vortex dynamics and relative equilibria}

In potential flow theory, an irrotational or point vortex is an idealized flow element that models circulating flow [15]. Since the flow at the center of a point vortex is undefined, it is called a flow singularity, much like a source or sink. Let $z_{\alpha}=x_{\alpha}+i y_{\alpha}$ represent the position of vortex $\alpha \in\{1, \ldots, N\}$ in the complex plane and $\gamma_{\alpha}>0$ be its strength, which determines the magnitude of the surrounding velocity field. The $N$-vortex system is Hamiltonian with [15]

$$
\begin{aligned}
& \gamma_{\alpha} \dot{x}_{\alpha}=\frac{\partial \mathcal{H}}{\partial y_{\alpha}}=-\frac{1}{2 \pi} \sum_{\beta \neq \alpha}^{N} \gamma_{\alpha} \gamma_{\beta} \frac{y_{\alpha}-y_{\beta}}{\left|z_{\alpha}-z_{\beta}\right|^{2}} \\
& \gamma_{\alpha} \dot{y}_{\alpha}=-\frac{\partial \mathcal{H}}{\partial x_{\alpha}}=\frac{1}{2 \pi} \sum_{\beta \neq \alpha}^{N} \gamma_{\alpha} \gamma_{\beta} \frac{x_{\alpha}-x_{\beta}}{\left|z_{\alpha}-z_{\beta}\right|^{2}},
\end{aligned}
$$

where the Hamiltonian $\mathcal{H}$ is

$$
\mathcal{H}=-\frac{1}{4 \pi} \sum_{\alpha=1}^{N} \sum_{\beta \neq \alpha}^{N} \gamma_{\alpha} \gamma_{\beta} \log \left|z_{\alpha}-z_{\beta}\right| .
$$


We elect to use complex variables for compactness and the overbar $\overline{(\cdot)}$ to denote complex conjugation. The selection of $\gamma_{k}>0$ ensures all vortices have the same signed circulation strength, preventing the possibility of vortex collapse. The Hamiltonian dynamics (1) and (2) are equivalent to [12],[15]

$$
\gamma_{\alpha} \dot{z}_{\alpha}=-2 i \frac{\partial \mathcal{H}}{\partial \bar{z}_{\alpha}}=\frac{i}{2 \pi} \sum_{\beta \neq \alpha}^{N} \gamma_{\alpha} \gamma_{\beta} \frac{z_{\alpha}-z_{\beta}}{\left|z_{\alpha}-z_{\beta}\right|^{2}},
$$

where the partial derivative operators are [21]

$$
\frac{\partial}{\partial z_{\alpha}} \triangleq \frac{1}{2}\left(\frac{\partial}{\partial x_{\alpha}}-i \frac{\partial}{\partial y_{\alpha}}\right) \text { and } \frac{\partial}{\partial \bar{z}_{\alpha}} \triangleq \frac{1}{2}\left(\frac{\partial}{\partial x_{\alpha}}+i \frac{\partial}{\partial y_{\alpha}}\right) .
$$

Let $\Gamma=\sum_{\alpha=1}^{N} \gamma_{\alpha}$ denote the total vorticity. In addition to $\mathcal{H}$, the $N$-vortex system conserves the center of vorticity $\mathcal{C}=\Gamma^{-1} \sum_{\alpha=1}^{N} \gamma_{\alpha} z_{\alpha}$ and the angular impulse $\mathcal{S}=\sum_{\alpha=1}^{N} \gamma_{\alpha}\left|z_{\alpha}\right|^{2}$ [15]. Note, these quantities are analogous to the center of mass and moment of inertia, respectively, in a point-mass system. Point vortices have the following relations to other flow singularities: replacing real circulation strength $\gamma_{\alpha}$ with an imaginary circulation strength $i \gamma_{\alpha}$ with $\gamma_{\alpha}>0$ (resp. $\left.\gamma_{\alpha}<0\right)$ produces a sink (resp. source); a complex circulation strength $\gamma_{\alpha}$ yields a spiral vortex [15].

Although the Hamiltonian dynamics of $N$ vortices in the plane yield chaotic trajectories [1], Chen et al. [1] show that adding dissipation stabilizes relative equilibria, which are geometrical configurations of vortices not fixed in an inertial frame [15]. Here we provide a Lyapunov analysis of the $N$-vortex dynamics with dissipation, making use of the conservation of angular impulse $\mathcal{S}=\sum_{\alpha=1}^{N} \gamma_{\alpha}\left|z_{\alpha}\right|^{2}$ in the construction of a Lyapunov function. We utilize the dissipative vortex dynamics for controlling the trajectories of actuated vortices in Section 4 .

Chen et al. [1] show that relative-equilibrium configurations of the vortex dynamics (4) are identical to the relative equilibria of the augmented system

$$
\dot{z}_{\alpha}=\frac{i}{2 \pi} \sum_{\beta \neq \alpha}^{N} \gamma_{\beta} \frac{z_{\alpha}-z_{\beta}}{\left|z_{\alpha}-z_{\beta}\right|^{2}}+\mu\left(\frac{1}{2 \pi} \sum_{\beta \neq \alpha}^{N} \gamma_{\beta} \frac{z_{\alpha}-z_{\beta}}{\left|z_{\alpha}-z_{\beta}\right|^{2}}-\omega z_{\alpha}\right),
$$

where $\mu>0$ repesents a gain that governs the rate of convergence to the equilibrium configuration. Setting $\mu=0$ in (5) yields the standard Hamiltonian vortex dynamics [1]; the significance of including $\mu>0$ is that the solutions of (5) converge asymptotically to stable relative equilibria of (4), which are rotating vortex configurations. Chen et al. refer to (5) as a phenomenological model; it represents dynamics that aggregate the particles into crystalline patterns [1]. Note that when a relative equilibrium is attained, the dissipative term is zero and the unmodified vortex dynamics are restored [1].

Let $\mathcal{R}$ be a rotating reference frame with angular rate $\omega$ relative to inertial frame $\mathcal{I}$, and consider the coordinate change $z_{\alpha}=\xi_{\alpha} \exp (i \omega t)$. By the chain 
rule, $\frac{\partial \mathcal{H}}{\partial \bar{z}_{\alpha}}=\frac{\partial \mathcal{H}}{\partial \bar{\xi}_{\alpha}} \exp (i \omega t)$ and $(4)$ becomes

$$
\gamma_{\alpha} \dot{\xi}_{\alpha}=-2 i \frac{\partial \mathcal{H}}{\partial \bar{\xi}_{\alpha}}-i \omega \gamma_{\alpha} \xi_{\alpha}
$$

The equilibria in $\mathcal{R}$ yield the following conditions for relative equilibria in $\mathcal{I}$ :

$$
\frac{\partial \mathcal{H}}{\partial \bar{\xi}_{\alpha}}+\frac{\omega}{2} \gamma_{\alpha} \xi_{\alpha}=0, \quad \alpha=1, \ldots, N .
$$

Note, these conditions are invariant under transformation back to $z_{\alpha}$, i.e.,

$$
\frac{\partial \mathcal{H}}{\partial \bar{z}_{\alpha}}+\frac{\omega}{2} \gamma_{\alpha} z_{\alpha}=\frac{\partial \mathcal{H}}{\partial \bar{z}_{\alpha}}+\frac{\omega}{2} \frac{\partial \mathcal{S}}{\partial \bar{z}_{\alpha}}=0
$$

The key observation is that (5) may be written in terms of the angular impulse $\mathcal{S}$, which is conserved by the unmodified dynamics, i.e.,

$$
\gamma_{\alpha} \dot{z}_{\alpha}=-2 i \frac{\partial \mathcal{H}}{\partial \bar{z}_{\alpha}}-2 \mu\left(\frac{\partial \mathcal{H}}{\partial \bar{z}_{\alpha}}+\frac{\omega}{2} \frac{\partial \mathcal{S}}{\partial \bar{z}_{\alpha}}\right) .
$$

The dynamics (9) consist of a Hamiltonian term and a gradient term. The gradient term suggests a Lyapunov function for asymptotic stability arguments, provided collisions between vortices do not occur. Consider the candidate Lyapunov function

$$
V=\mathcal{H}+\frac{\omega}{2} \mathcal{S}
$$

whose dynamics along solutions of (9) are

$$
\begin{aligned}
\dot{V} & =\sum_{\alpha} \frac{\partial V}{\partial z_{\alpha}} \dot{z}_{\alpha}+\frac{\partial V}{\partial \bar{z}_{\alpha}} \dot{\bar{z}}_{\alpha} \\
& =\sum_{\alpha} \frac{2 \omega}{\gamma_{\alpha}} \operatorname{Re}\left(-i \frac{\partial \mathcal{H}}{\partial \bar{z}_{\alpha}} \frac{\partial \mathcal{S}}{\partial z_{\alpha}}\right)-\frac{4 \mu}{\gamma_{\alpha}}\left|\frac{\partial \mathcal{H}}{\partial \bar{z}_{\alpha}}+\frac{\omega}{2} \frac{\partial \mathcal{S}}{\partial \bar{z}_{\alpha}}\right|^{2} .
\end{aligned}
$$

By plugging in for $\mathcal{H}$ and $\mathcal{S}$ and making use of $\operatorname{Im}\left(\sum_{\alpha} \sum_{\beta \neq \alpha} z_{\beta} \bar{z}_{\alpha}\right)=0$, the first term vanishes, leaving

$$
\dot{V}=-\sum_{\alpha} \frac{4 \mu}{\gamma_{\alpha}}\left|\frac{\partial \mathcal{H}}{\partial \bar{z}_{\alpha}}+\frac{\omega}{2} \frac{\partial \mathcal{S}}{\partial \bar{z}_{\alpha}}\right|^{2} \leq 0 .
$$

This observation leads to the following proposition.

Proposition 1. Suppose $\gamma_{\alpha}>0$ and $z_{\alpha}(t)$ is a collision-free, bounded trajectory of (9) for all $\alpha=1, \ldots, N$. Then $z_{\alpha}(t)$ asymptotically converges to a relative equilibrium of the vortex dynamics given by (4).

Proof. By assumption, $z_{\alpha}(t)$ is collision-free and bounded. Hence, $V$ is welldefined for all time and there exists a compact set within which the trajectories reside for all time. $\dot{V}$ is negative semi-definite and zero only when (8) is satisfied $\forall \alpha=1, \ldots, N$. The invariance principle [7] stipulates that the trajectories asymptotically converge to the largest invariant set for which condition (8) holds; this set contains the rotating relative equilibria of (4). 


\section{Active singularities for motion planning}

The vortex dynamics with dissipation (5) are useful for stabilizing relative equilibria. We now exploit this property to create a novel motion-planning paradigm based on virtual spiral vortices, which are singularities that add dissipation to a point vortex system. Spiral vortices have complex circulation strength because of the collocation of a vortex and a source or sink [15]. In this framework, virtual vortices generate control vector fields that are added to the drift vector field associated with the fluid flow. The control inputs to the system are the singularity strengths.

Suppose the trajectories of $P$ vehicles are generated by integrating their dynamic interactions with $N$ actual vortices and $M$ virtual (spiral) vortices. Let the actual vortices be located at $z_{\alpha}$ for $\alpha \in\{1, \ldots, N\}$. Append the virtual vortex locations $z_{\alpha}$ for $\alpha \in\{N+1, \ldots, N+M\}$ and vehicle locations $z_{\alpha}$ for $\alpha \in\{N+M+1, \ldots, N+M+P\}$ to form the state vector $z_{\alpha} \in \mathbb{C}^{N+M+P}$. Let $\Gamma_{\beta}$ be the (possibly complex) circulation of vortex $\beta=1, \ldots, N+M$. The dynamics of the actual and virtual vortices in the active singularity system are

$$
\dot{z}_{\alpha}=\frac{i}{2 \pi} \sum_{\beta \neq \alpha}^{N+M} \Gamma_{\beta} a_{\alpha, \beta} \frac{z_{\alpha}-z_{\beta}}{\left|z_{\alpha}-z_{\beta}\right|^{2}}, \quad \alpha=1, \ldots, N+M,
$$

where the sum is taken over $N+M$ to account for all singularities in the system. The interaction topology

$$
a_{\alpha, \beta}=\left\{\begin{array}{ll}
0, & \text { if } \alpha=\beta \text { or }(\alpha \leq N \text { and } \beta>N) \\
1, & \text { otherwise }
\end{array},\right.
$$

enforces the natural dynamics between actual vortices, whereas each virtual vortex evolves under the combined influence of the actual vortices and the other virtual vortices. The vehicle dynamics $\dot{z}_{\alpha}$ for $\alpha=N+M+1, \ldots, N+M+P$ depend on the association between virtual vortices and vehicles. The association may be one-to-one, multiple-to-one, involve a fixed virtual vortex, or be a mixed variant of these strategies. In the remainder of this paper, we assume that virtual vortices are collocated with vehicles under a one-to-one association.

View the complex circulations $\left\{\Gamma_{N+1}, \ldots, \Gamma_{N+M}\right\}$ associated with the virtual (spiral) vortices as control inputs; the problem is to characterize and stabilize the desired solutions of (11). Note that because it is necessary to have more than a single virtual vortex for controllability, the circulation strength $\Gamma_{\beta}$ of virtual vortex $\beta$ only influences virtual vortex $\beta$ indirectly. When $\operatorname{Re}\left\{\Gamma_{\beta}\right\}=0$ and $\operatorname{Im}\left\{\Gamma_{\beta}\right\} \neq 0$, the active singularity $\beta$ has radial flow only [15] (i.e., it is a source for $\operatorname{Im}\left\{\Gamma_{\beta}\right\}<0$ or a sink for $\left.\operatorname{Im}\left\{\Gamma_{\beta}\right\}>0\right)$. When $\operatorname{Re}\left\{\Gamma_{\beta}\right\} \neq 0$ and $\operatorname{Im}\left\{\Gamma_{\beta}\right\}=0$, the active singularity $\beta$ represents an irrotational vortex. When $\Gamma_{\beta}=0, \beta$ behaves as a passive (drifting) particle. Observe that if each vehicle is assigned to a virtual vortex, (11) is identical to (5) with $\Gamma_{\beta}=\gamma_{\beta}-i \mu \gamma_{\beta}$ for spiral vortices and a fixed sink of strength $2 \pi \mu \omega\left|z_{\alpha}\right|^{2}$ at the origin. The sink at the origin serves only to center the relative equilibrium at the origin. 


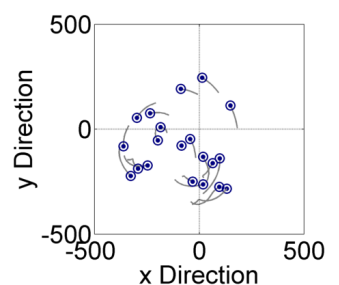

(a) $t=1 \mathrm{sec}$

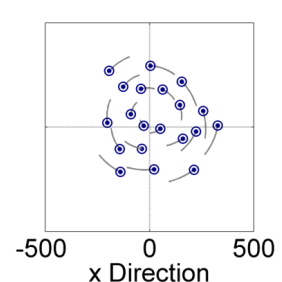

(b) $t=10 \mathrm{sec}$

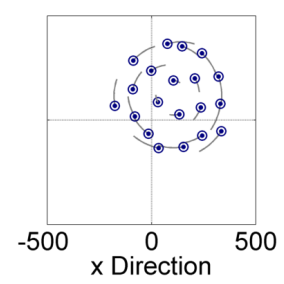

(c) $t=20 \mathrm{sec}$

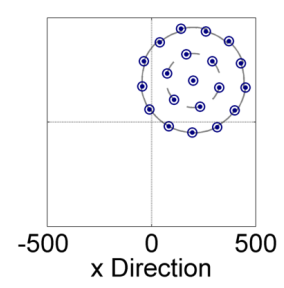

(d) $\mathrm{t}=100 \mathrm{sec}$

Fig. 1: Simulating convergence to a relative equilibrium centered at $\mathcal{C}_{0}=200+200 i$

In fact, one may control the center of vorticity $\mathcal{C}$ (correspondingly, the center of the relative equilibrium) to be at a point $\mathcal{C}_{0} \in \mathbb{C}$ away from the origin. Define the shifted angular impulse

$$
\mathcal{S}^{\prime}=\sum_{\alpha=1}^{N} \gamma_{\alpha}\left|z_{\alpha}-\mathcal{C}_{0}\right|^{2}
$$

and the modified Lyapunov function

$$
V^{\prime}=\mathcal{H}+\frac{\omega}{2} \mathcal{S}^{\prime}
$$

The following corollary to Proposition 1 represents a preliminary design consideration for virtual vortex control.

Corollary 1. Suppose $\gamma_{\alpha}>0$ and $z_{\alpha}(t)$ is a collision-free, bounded trajectory of (9) with $\mathcal{S}$ replaced by $\mathcal{S}^{\prime}$ for all $\alpha=1, \ldots, N$. Then $z_{\alpha}(t)$ asymptotically converges to a relative equilibrium of the vortex dynamics given by (4) and centered at $\mathcal{C}_{0}$.

Proof. Substituting $\mathcal{S}^{\prime}$ into (9) in place of $\mathcal{S}$, using the Lyapunov function (14), and following the approach of Prop. 1 shows that solutions converge to the largest invariant set in which (8) is satisfied with $\mathcal{S}$ replaced by $\mathcal{S}^{\prime}$. This implies $\mathcal{C}=\mathcal{C}_{0}$ because (after summing the invariance condition over all $\alpha$ ), $\sum_{\alpha=1}^{N} \gamma_{\alpha}\left(z_{\alpha}-\mathcal{C}_{0}\right)=$ $\Gamma\left(\mathcal{C}-\mathcal{C}_{0}\right)=0$.

Figure 1 illustrates the time evolution of virtual vortices from a random initial arrangement to a rotating relative equilibrium centered at $\mathcal{C}_{0}=200+200 i$.

We now consider the actual-plus-virtual vortex system. Suppose the actual vortices interact according to their own natural dynamics (4) and the virtual vortices have dynamics

$$
\gamma_{\alpha} \dot{z}_{\alpha}=-2 i \frac{\partial \mathcal{H}_{c}}{\partial \bar{z}_{\alpha}}-2 \mu\left(\frac{\partial \mathcal{H}_{c}}{\partial \bar{z}_{\alpha}}+\frac{w}{2} \gamma_{\alpha} z_{\alpha}\right),
$$

for $\alpha=N+1, \ldots, N+M$, where

$$
\mathcal{H}_{c}=-\frac{1}{2 \pi} \sum_{\alpha}^{N} \sum_{\substack{N+1 \\ \beta \neq \alpha}}^{N+M} \gamma_{\alpha} \gamma_{\beta} \log \left|z_{\alpha}-z_{\beta}\right|-\frac{1}{4 \pi} \sum_{\substack{N+1 \\ \alpha}}^{N+M} \sum_{\substack{N+1 \\ \beta \neq \alpha}}^{N+M} \gamma_{\alpha} \gamma_{\beta} \log \left|z_{\alpha}-z_{\beta}\right| .
$$


For $\mu=0$, it can be shown that the virtual vortices conserve $\mathcal{H}_{c}$ even when interacting with the actual vortices (omitted for brevity). In the active singularity system, the dynamics (15) asymptotically stabilize relative equilibrium configurations of the virtual vortices in the presence of actual vortices, which are themselves in relative equilibrium. The formulation (15) of the path-planning problem gives rise to a Lyapunov-based control design in which one selects the dissipative terms to asymptotically stabilize a desired vehicle configuration for environmental sampling. In the following result, we make use of the conserved quantity $\mathcal{H}_{c}$ to suggest a candidate Lyapunov function.

Define the candidate Lyapunov function

$$
V_{c}=\mathcal{H}_{c}+\frac{\omega}{2} \mathcal{S}_{c},
$$

where the angular impulse of the virtual vortices $\mathcal{S}_{c}$ is

$$
\mathcal{S}_{c}=\sum_{\alpha=N+1}^{N+M} \gamma_{\alpha}\left|z_{\alpha}\right|^{2} .
$$

The actual vortices contribute time-varying terms to the virtual vortex dynamics. However, in the rotating frame $\mathcal{R}$, the actual vortices appear fixed. Hence, changing coordinates yields

$$
\dot{V}_{c}=\sum_{\alpha=N+1}^{N+M} \frac{-4 \mu}{\gamma_{\alpha}}\left|\frac{\partial \mathcal{H}_{c}}{\partial \xi_{\alpha}}+\frac{\omega}{2} \frac{\partial \mathcal{S}_{c}}{\partial \xi_{\alpha}}\right|^{2} \leq 0,
$$

leading to the following proposition.

Proposition 2. Suppose $\gamma_{\alpha}>0$ and $z_{\alpha}(t)$ is a collision-free, bounded trajectory of the virtual vortex dynamics (15) for all $\alpha=N+1, \ldots, N+M$, in the presence of actual point-vortices in relative equilibrium. Then $z_{\alpha}(t)$ asymptotically converges to a relative equilibrium configuration of the actual-plus-virtual vortex system.

Proof. Proceeding in the same manner as Prop. 1 and applying LaSalle's invariance principle [7] shows the virtual vortices converge to the largest invariant set that satisfies condition $(7)$ with $\mathcal{H}$ replaced by $\mathcal{H}_{c}$, which is a relative equilibrium configuration fixed in rotating frame $\mathcal{R}$.

Figure 2(a) illustrates the stabilization of relative equilibrium for $M=20$ virtual vortices (blue) under the dynamics (15) in the presence of an actual vortex pair (green). Observe that the virtual vortices surround the origin and naturally separate into a rotating formation. The size and shape of the formation depend on the virtual and actual vortex strengths $\gamma_{\alpha}$ and the initial conditions. Figures 2(b)-(e) display simulation results of a set of weaker virtual vortices achieving a different configuration. Note that during convergence to the relative equilibrium, virtual vortices move along the flow streamlines while also interacting with each other. This choice of circulation strength yields two separate sampling groups located within the invariant regions of the flow generated by the actual vortex pair. 


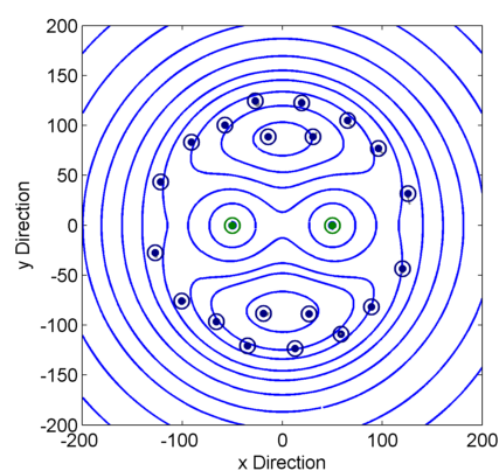

(a)

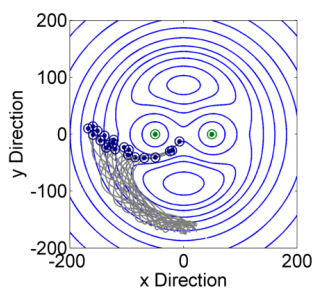

(b) $\mathrm{t}=10 \mathrm{sec}$

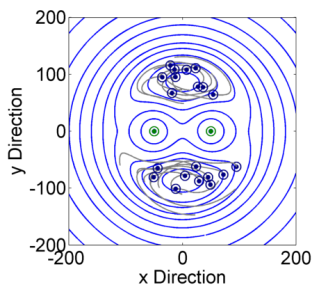

(d) $\mathrm{t}=50 \mathrm{sec}$

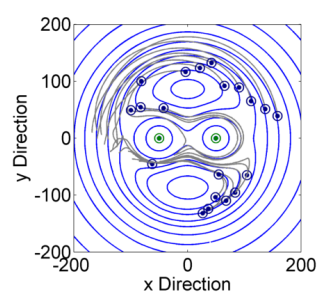

(c) $t=25 \mathrm{sec}$

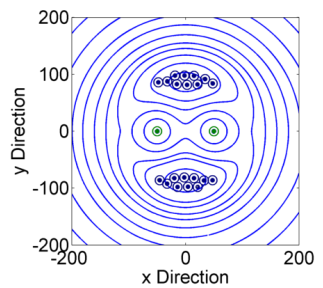

(e) $\mathrm{t}=150 \mathrm{sec}$

Fig. 2: (a) Actual-plus-virtual vortex system in relative equilibrium in frame $\mathcal{R}$; (b)-(e) Simulation of weaker virtual vortices undergoing relative equilibrium stabilization in the presence of an actual vortex pair

\section{Adaptive control design}

The use of Lyapunov analysis to inform the selection of a parameter update law is a common theme in adaptive control [22]. We employ this technique for the selection of a circulation-strength update law in the following example application of the path-planning methodology. Preliminary results show convergence to a desired level set $\mathcal{H}_{c}^{d}$ of the controlled Hamiltonian $\mathcal{H}_{c}$.

Consider the candidate Lyapunov function

$$
V_{c}^{d}=\frac{1}{2}\left(\mathcal{H}_{c}-\mathcal{H}_{c}^{d}\right)^{2}
$$

For simplicity of exposition and also to avoid virtual vortices having opposite signed circulation strengths, we restrict $\gamma_{k}=\gamma>0$ for this example. Along trajectories of (15), the Lyapunov function (18) yields

$$
\begin{aligned}
\dot{V}_{c}^{d} & =\left(\mathcal{H}_{c}-\mathcal{H}_{c}^{d}\right)\left[\sum_{\alpha=N+1}^{N+M} \frac{\partial \mathcal{H}_{c}}{\partial \xi_{\alpha}} \dot{\xi}_{\alpha}+\frac{\partial \mathcal{H}_{c}}{\partial \bar{\xi}_{\alpha}} \dot{\bar{\xi}}_{\alpha}+\frac{\partial \mathcal{H}_{c}}{\partial \gamma} \dot{\gamma}\right] \\
& =\left(\mathcal{H}_{c}-\mathcal{H}_{c}^{d}\right)\left[\sum_{\alpha=N+1}^{N+M} \frac{-4 \mu}{\gamma}\left|\frac{\partial \mathcal{H}_{c}}{\partial \bar{\xi}_{\alpha}}\right|^{2}-\frac{2 \omega}{\gamma}\left(\mu \operatorname{Re}\left\{\frac{\partial \mathcal{H}_{c}}{\partial \bar{\xi}_{\alpha}} \frac{\partial \mathcal{S}_{c}}{\partial \xi_{\alpha}}\right\}+\operatorname{Im}\left\{\frac{\partial \mathcal{H}_{c}}{\partial \bar{\xi}_{\alpha}} \frac{\partial \mathcal{S}_{c}}{\partial \xi_{\alpha}}\right\}\right)+\frac{\partial \mathcal{H}_{c}}{\partial \gamma} \dot{\gamma}\right] .
\end{aligned}
$$




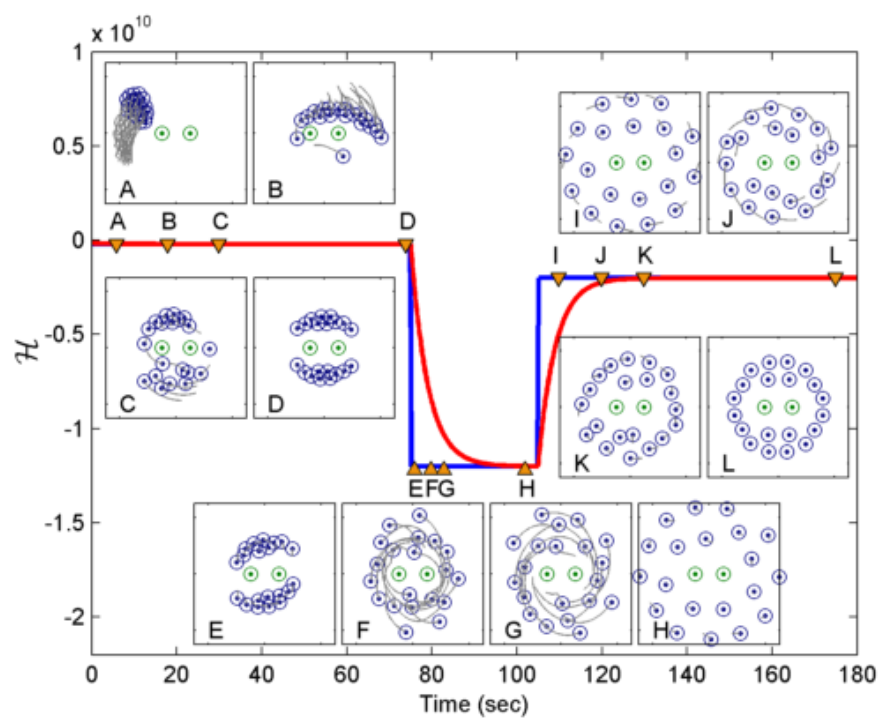

Fig. 3: Formations of virtual vortices under adaptive circulation control

The following choice of adaptation rate $\dot{\gamma}$ provides negative semi-definiteness of $\dot{V}_{c}^{d}$. In particular, choosing

$$
\begin{aligned}
\dot{\gamma}= & \left(\frac{\partial \mathcal{H}_{c}}{\partial \gamma}\right)^{-1}\left[-\sum_{\alpha=N+1}^{N+M}\left(\frac{-4 \mu}{\gamma}\left|\frac{\partial \mathcal{H}_{c}}{\partial \bar{\xi}_{\alpha}}\right|^{2}-\right.\right. \\
& \left.\left.\frac{2 \omega}{\gamma}\left(\mu \operatorname{Re}\left\{\frac{\partial \mathcal{H}_{c}}{\partial \bar{\xi}_{\alpha}} \frac{\partial \mathcal{S}_{c}}{\partial \xi_{\alpha}}\right\}+\operatorname{Im}\left\{\frac{\partial \mathcal{H}_{c}}{\partial \bar{\xi}_{\alpha}} \frac{\partial \mathcal{S}_{c}}{\partial \xi_{\alpha}}\right\}\right)\right)-K\left(\mathcal{H}_{c}-\mathcal{H}_{c}^{d}\right)\right]
\end{aligned}
$$

results in $\dot{V}_{c}^{d} \leq-K\left(\mathcal{H}_{c}-\mathcal{H}_{c}^{d}\right)^{2}$, where $\dot{V}_{c}^{d}=0$ only when the invariance condition $\mathcal{H}_{c}-\mathcal{H}_{c}^{d} \equiv 0$ holds.

Choosing the circulation of the virtual vortices to have the same sign as the actual vortices prevents the $\left(\partial \mathcal{H}_{c} / \partial \gamma\right)^{-1}$ term in (19) from becoming singular as long as $\left|z_{\alpha}-z_{\beta}\right|>1$ for all pairs $\alpha, \beta$ due to the $\log (\cdot)$ terms in $\partial \mathcal{H}_{c} / \partial \gamma$. If is $\gamma$ bounded, which remains to be shown, the invariance condition implies that $\mathcal{H}_{c}$ converges to $\mathcal{H}_{c}^{d}$, but it enforces nothing else about the configuration. The virtual vortices interact according to (15) in a rotating frame. A sufficient condition for maintaining a constant $\mathcal{H}_{c}$ value is for the second term in (15) to vanish, since the virtual vortices conserve $\mathcal{H}_{c}$ when $\mu=0$. Although $\mu \neq 0$, the second term can still vanish if the rotating equilibrium condition is satisfied. In fact, virtual vortices converge to relative equilibrium configurations rotating at angular rate $\omega$, as illustrated in the following numerical experiment.

Figure 3 displays a numerical simulation of the adaptive control algorithm for $M=20$ virtual vortices in the presence of two actual vortices. Figure 3 shows the desired $\mathcal{H}_{c}^{d}$ signal (blue) and actual $\mathcal{H}_{c}$ signal (red). Inset plots are 
snapshots of the virtual vortices (blue) in the presence of the actual vortices (green) in frame $\mathcal{R}$. Virtual vortices interact with each other and the actual vortices during movement to the relative equilibrium. Additionally, the vortices form concentric disk formations influenced by the physical flow environment. For the $\mathcal{H}_{c}^{d}$ values chosen, the virtual vortex disk-like formations enclose the actual vortices, with disk size controlled by the specified $\mathcal{H}_{c}^{d}$ value. The locations of the virtual vortices in each configuration do not necessarily have exact symmetry, because the configuration in $\mathcal{H}_{c}^{d}$ is not unique.

\section{Conclusion}

This paper describes a distributed motion-planning paradigm for sampling applications in vortical flow environments. This paradigm enables theoretically justified control laws whose stability properties are ensured by virtue of a Lyapunovbased design that leverages the underlying Hamiltonian structure of the vortex dynamics. The approach employs fluid-like interactions between physical and virtual vortices based on the Hamiltonian dynamics. Gradient dynamics cause the virtual vortices to converge to relative equilibria configurations. We provide an adaptive control to regulate a collection of virtual vortices. Refinement and extension of this methodology to account for vehicle-specific constraints, timevarying and uncertain flows, track feasibility, and communication delays are all subjects of ongoing work.

Acknowledgments. The authors of this work acknowledge valuable discussions with Kayo Ide, Levi DeVries, Nitin Sydney and Daigo Shishika, and support by the National Science Foundation under Award No. CMMI-1362837.

\section{References}

1. Chen, Y., Kolokolnikov, T., Zhirov, D.: Collective behaviour of large number of vortices in the plane. Proc. R. Soc. A 469, 1-12 (2013)

2. Das, J., Py, F., Maughan, T., O'Reilly, T., Messié, M., Ryan, J., Sukhatme, G.S., Rajan, K.: Coordinated sampling of dynamic oceanographic features with underwater vehicles and drifters. Int. J. Robotics Res. 31(5), 626-646 (2012)

3. DeVries, L., Paley, D.A.: Multivehicle control in a strong flowfield with application to hurricane sampling. J. Guidance Control Dyn. 35(3), 794-806 (2012)

4. Frew, E.W., Lawrence, D.A., Morris, S.: Coordinated standoff tracking of moving targets using Lyapunov guidance vector fields. J. Guidance Control Dyn. 31(2), 290-306 (2008)

5. Hoffman, M.J., Miyoshi, T., Haine, T.W.N., Ide, K., Brown, C.W., Murtugudde, R.: An advanced data assimilation system for the Chesapeake Bay: Performance evaluation. J. Atmos. Ocean. Technol. 29(10), 1542-1557 (2012)

6. Hsieh, M.A., Forgoston, E., Mather, T.W., Schwartz, I.B.: Robotic manifold tracking of coherent structures in flows. In: IEEE Int. Conf. Robotics and Automation (ICRA). pp. 4242 - 4247 (2012) 
7. Khalil, H.: Nonlinear systems. Prentice Hall, Upper Saddle River N.J., 3rd edn. (2002)

8. Koszalka, I.: Mesoscale vortices, Lagrangian transport and marine ecosystem dynamics. Ph.D. thesis, Politecnico Di Torino (2008)

9. Lermusiaux, P.F.J., Lolla, T., Haley Jr., P.J., Yigit, K., Ueckermann, M.P., Sondergaard, T., Leslie, W.G.: Science of autonomy: Time-optimal path planning and adaptive sampling for swarms of ocean vehicles. In: Curtin, T. (ed.) Springer Handbook Ocean Eng. Auton. Ocean Veh. Subsystems Control. Springer-Verlag (2013)

10. Lolla, T., Ueckermann, M.P., Yigit, K., Haley Jr., P.J., Lermusiaux, P.F.J.: Path planning in time dependent flow fields using level set methods. In: IEEE Int. Conf. Robotics and Automation (ICRA). pp. 166-173 (2012)

11. Mallory, K., Hsieh, M.A., Forgoston, E., Schwartz, I.B.: Distributed allocation of mobile sensing swarms in gyre flows. Nonlinear Processes in Geophysics 20(5), 657-668 (2013)

12. Marsden, J.E., Ratiu, T.S.: Introduction to mechanics and symmetry, vol. 48. Amer. Inst. of Physics, New York (1995)

13. Melet, A., Verron, J., Brankart, J.M.: Potential outcomes of glider data assimilation in the Solomon Sea: Control of the water mass properties and parameter estimation. J. Marine Sys. 94, 232-246 (2012)

14. Mourre, B., Alvarez, A.: Benefit assessment of glider adaptive sampling in the Ligurian Sea. Deep Sea Res. Part I Ocean. Res. Papers 68, 68-78 (2012)

15. Newton, P.: The N-vortex problem: Analytical techniques. Springer, New York (2001)

16. Paley, D., Zhang, F., Leonard, N.: Cooperative control for ocean sampling: The Glider Coordinated Control System. IEEE Trans. Control Sys. Tech. (1063-6536), 1-10 (2008)

17. Pazos, M.: The GDP drifter data assembly center (DAC): Hurricane drifter array, NOAA AOML, http://www.aoml.noaa.gov/phod/dac/dacdata.php

18. Ramp, S., Davis, R., Leonard, N., Shulman, I., Chao, Y., Robinson, A., Marsden, J., Lermusiaux, P., Fratantoni, D., Paduan, J., Chavez, F., Bahr, F., Liang, S., Leslie, W., Li, Z.: Preparing to predict: The second Autonomous Ocean Sampling Network (AOSN-II) experiment in the Monterey Bay. Deep Sea Res. Part II: Topical Studies Ocean. 56(3-5), 68-86 (2009)

19. Rudnick, D.L., Cole, S.T.: On sampling the ocean using underwater gliders. J. Geophys. Res. 116(C08010), 1-12 (2011)

20. Smith, R.N., Schwager, M., Smith, S.L., Jones, B.H., Rus, D., Sukhatme, G.S.: Persistent ocean monitoring with underwater gliders: Adapting sampling resolution. J. Field Robotics 28(5), 714-741 (2011)

21. Sorber, L., Barel, M., Lathauwer, L.: Unconstrained optimization of real functions in complex variables. SIAM J. Optim. 22(3), 879-898 (2012)

22. Spong, M., Hutchinson, S., and Vidyasagar, M.: Robot modeling and control. John Wiley \& Sons, Hoboken, NJ (2006)

23. Zhang, D., Colburn, C., Bewley, T.: Estimation and adaptive observation of environmental plumes. In: Amer. Control Conf. pp. 4281-4286. San Francisco, CA (2011) 